\title{
A Simple Method for $N$-Phenoxyethylation of Anilines
}

\author{
G. P. Romanelli, J. L. Jios, O. Guaymas, R. Piovoso and J. C. Autino \\ LADECOR, Departamento de Química, Facultad de Ciencias Exactas, Universidad Nacional de La \\ Plata. Calles 47 y 115, B1900AJL La Plata, Argentina \\ E-mail: jautino@quimica.unlp.edu.ar
}

\begin{abstract}
We wanted to search for new reaction conditions to prepare the title compounds, to be checked later in novel syntheses of heterocyclic compounds. To the best of our knowledge, there was no report in the literature of any well-established method for the preparation of $N$-(2-phenoxyethyl)anilines 1.
\end{abstract}

The scarce previously reported preparations involved large excesses of some starting materials, relatively high temperatures and long reaction times $[1,2]$. We have recently reported a general procedure for that preparation [3] although at that stage only moderate yields were obtained. We describe here a better and simpler procedure for achieving not only compounds $\mathbf{1}$ in good yields, but also for extending the scope of the reaction to the synthesis of the related bis- $N$-(2-phenoxy-ethyl)anilines $\mathbf{2}$. In order to avoid $\beta$-elimination reactions in molecules bearing a phenoxyethyl group, the reaction was carried out precluding strong acidic or basic media, see Scheme.

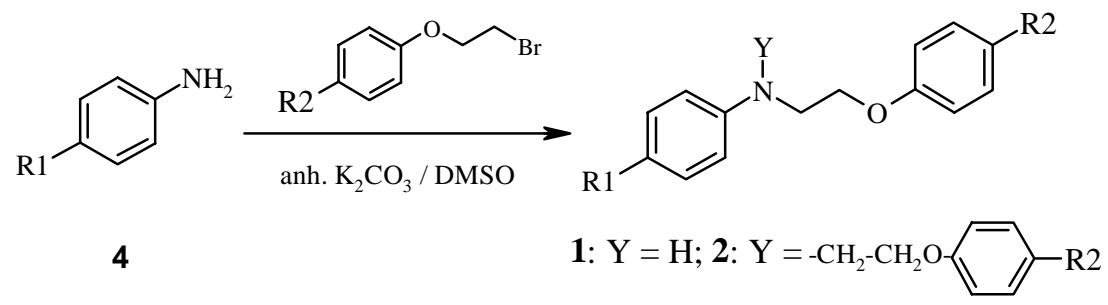

Scheme. N-phenoxyethylation reaction of anilines.

Dependimng on the product desired, 1-bromophenoxyethanes (3) or anilines (4) were used in molar excess. To prepare mono- $N$-(2-phenoxyethyl)anilines (1) the reagent 4 was used in excess, yielding 70$80 \%$ (see Table), whereas an excess of $\mathbf{3}$ lead to 2 with yields ranging in 50-70\%.

Reaction conditions involve typically $90^{\circ} \mathrm{C}$, DMSO as solvent and anhydrous $\mathrm{K}_{2} \mathrm{CO}_{3}$ as the base. Yields are substantially improved as compared with those already obtained using triethylamine [3]. New compounds $\mathbf{2 b}$ and $\mathbf{2 c}$ gave satisfactory analytical and spectroscopic data. 
Table. Selected examples of N-(2-phenoxyethyl)anilines $\mathbf{1}$ and $\mathbf{2}$.

\begin{tabular}{c|ccc} 
Compound & R1 & R2 & \% Yield \\
\hline 1a & $\mathrm{H}$ & $\mathrm{H}$ & 75 \\
$\mathbf{1 b}$ & $\mathrm{H}$ & $\mathrm{OMe}$ & 72 \\
$\mathbf{1 c}$ & $\mathrm{H}$ & $\mathrm{NO}_{2}$ & 71 \\
$\mathbf{1 d}$ & $\mathrm{OMe}$ & $\mathrm{H}$ & 79 \\
$\mathbf{1 e}$ & $\mathrm{NO}_{2}$ & $\mathrm{H}$ & 58 \\
$\mathbf{2 a}$ & $\mathrm{H}$ & $\mathrm{H}$ & 55 \\
$\mathbf{2 b}$ & $\mathrm{H}$ & $\mathrm{Cl}$ & 59 \\
$\mathbf{2 c}$ & $\mathrm{Cl}$ & $\mathrm{H}$ & 63
\end{tabular}

\section{References and Notes}

1. Oki, M.; Mutai, K. Intramolecular N-H...O hydrogen bonding in N-( $\omega$-phenoxyalkyl)anilines. Spectrochim. Acta 1969, A25, 1941.

2. Mutai, K.; Tukada, H.; Nakagaki, R. Bifunctional Reactivity of the Nitrophenoxyl Group in Intramolecular Photoreactions. J. Org. Chem. 1991, 56, 4896.

3. Autino, J. C.; Bruzzone, L.; Romanelli, G. P.; Jios, J. L.; Ancinas, H. A. Substituted Nphenoxyethylanilines: preparation and acid-base properties evaluation by fluorescence spectrometry. Anales de Química, Intern. Ed. 1998, 94, 292. 